\title{
Some combinatorial identities via Stirling transform
}

\author{
Fouad Bounebirat ${ }^{1}$, Diffalah Laissaoui ${ }^{2}$ \\ and Mourad Rahmani ${ }^{3}$ \\ ${ }^{1}$ USTHB, Faculty of Mathematics \\ P. O. Box 32, El Alia 16111, Bab-Ezzouar, Algiers, Algeria \\ e-mail: bounebiratfouadeyahoo. fr \\ ${ }^{2}$ Dr Yahia Farès University of Médéa \\ Médéa 26000, Algeria \\ e-mail: laissaoui.diffalah74@gmail.com \\ ${ }^{3}$ USTHB, Faculty of Mathematics \\ P. O. Box 32, El Alia 16111, Bab-Ezzouar, Algiers, Algeria \\ e-mails: mrahmani@usthb.dz, rahmani.mouradegmail.com
}

Received: 28 January 2017

Accepted: 25 September 2018

\begin{abstract}
The aim of this paper is to present some results on the use of the generalized Stirling transform. First, we establish a generalization of a recent Guo-Qi's identity for Bell numbers. Finally, a new explicit formula for Euler numbers are given.
\end{abstract}

Keywords: Bell numbers, Lah numbers, Stirling transform.

2010 Mathematics Subject Classification: 11B73, 33C05.

\section{Introduction}

Following the usual notations (see [3]), the falling factorial $x^{\underline{n}}(x \in \mathbb{C})$ is defined by $x^{\underline{0}}=1$, $x^{\underline{n}}=x(x-1) \cdots(x-n+1)$ for $n>0$ and, the rising factorial denoted by $x^{\bar{n}}$, is defined by $x^{\bar{n}}=x(x+1) \cdots(x+n-1)$ with $x^{\overline{0}}=1$. The (signed) Stirling numbers of the first kind $s(n, k)$ are the coefficients in the expansion

$$
x^{\underline{n}}=\sum_{k=0}^{n} s(n, k) x^{k} .
$$


The Stirling numbers of the second kind, denoted by $\left\{\begin{array}{l}n \\ k\end{array}\right\}$, are the coefficients in the expansion

$$
x^{n}=\sum_{k=0}^{n}\left\{\begin{array}{l}
n \\
k
\end{array}\right\} x^{\underline{k}} .
$$

The Stirling numbers of the second kind $\left\{\begin{array}{l}n \\ k\end{array}\right\}$ count the number of ways to partition a set of $n$ elements into exactly $k$ nonempty subsets. The number of all partitions is the Bell number $B_{n}$, thus

$$
B_{n}=\sum_{k=0}^{n}\left\{\begin{array}{l}
n \\
k
\end{array}\right\}
$$

The polynomials

$$
B_{n}(x)=\sum_{k=0}^{n}\left\{\begin{array}{l}
n \\
k
\end{array}\right\} x^{k}
$$

are called Bell polynomials or exponential polynomials. The exponential generating functions are respectively

$$
\begin{gathered}
\sum_{n \geq k} s(n, k) \frac{z^{n}}{n !}=\frac{1}{k !}(\ln (1+z))^{k}, \\
\sum_{n \geq k}\left\{\begin{array}{l}
n \\
k
\end{array}\right\} \frac{z^{n}}{n !}=\frac{1}{k !}\left(e^{z}-1\right)^{k}
\end{gathered}
$$

and

$$
\sum_{n \geq 0} B_{n}(x) \frac{z^{n}}{n !}=\exp \left(x\left(e^{z}-1\right)\right) .
$$

Recently, the third author [6] developed a methodology for computing the Stirling transform and the inverse Stirling transform. More precisely, given a sequence $a_{m}:=a_{0, m}(m \geq 0)$, we construct an infinite matrix $\mathcal{S}:=\left(a_{n, m}\right)$ as follows:

1. The first row $a_{0, m}$ of the matrix is the initial sequence; the first column $b_{n}:=a_{n, 0}(n \geq 0)$ is called the final sequence, and each entry $a_{n, m}$ is given recursively by

$$
a_{n+1, m}=a_{n, m+1}+m a_{n, m} .
$$

2. Conversely, if we start with the final sequence, the matrix $\mathcal{S}$ can be recovered by the recursive relations

$$
a_{n, m+1}=a_{n+1, m}-m a_{n, m} .
$$

Theorem 1. [6] For $n, m \geq 0$, we have

$$
a_{n, m}=\sum_{k=0}^{m} s(m, k) b_{n+k}=\sum_{k=0}^{n}\left\{\begin{array}{l}
n+m \\
k+m
\end{array}\right\}_{m} a_{m+k} .
$$

Recall that the $r$-Stirling numbers of the second kind (see [1] for more details) $\left\{\begin{array}{l}n \\ k\end{array}\right\}_{r}$ count the number of partitions of a set of $n$ objects into exactly $k$ nonempty, disjoint subsets, such that the first $r$ elements are in distinct subsets. The exponential generating function is given by

$$
\sum_{n \geq k}\left\{\begin{array}{l}
n+r \\
k+r
\end{array}\right\}_{r} \frac{z^{n}}{n !}=\frac{1}{k !} e^{r z}\left(e^{z}-1\right)^{k} .
$$


Theorem 2. [6] Suppose that the initial sequence $a_{0, m+r}$ has the following exponential generating function $A_{r}(z)=\sum_{k \geq 0} a_{0, k+r} \frac{z^{k}}{k !}$. Then the sequence $\left\{a_{n, r}\right\}_{n}$ of the $r$-th columns of the matrix $\mathcal{S}$ has an exponential generating function $\mathcal{B}_{r}(z)=\sum_{n \geq 0} a_{n, r} \frac{z^{n}}{n !}$, given by

$$
B_{r}(z)=e^{r z} A_{r}\left(e^{z}-1\right) .
$$

Theorem 3. [6] Suppose that the final sequence $a_{n+r, 0}$ has the following exponential generating function $\mathcal{B}_{r}(z)=\sum_{k \geq 0} a_{k+r, 0} \frac{z^{k}}{k !}$. Then the sequence $\left\{a_{r, m}\right\}_{m}$ of the $r$-th rows of the matrix $\mathcal{S}$ has an exponential generating function $\mathcal{A}_{r}(z)=\sum_{m \geq 0} a_{r, m} \frac{z^{m}}{m !}$, given by

$$
\mathcal{A}_{r}(z)=\mathcal{B}_{r}(\ln (1+z))
$$

\section{On Guo-Qi's identity for Bell numbers}

The (unsigned) Lah numbers $\left\lfloor\begin{array}{l}n \\ k\end{array}\right\rfloor$ are the coefficients expressing rising factorials in terms of falling factorials

$$
x^{\bar{n}}=\sum_{k=0}^{n}\left\lfloor\begin{array}{l}
n \\
k
\end{array}\right\rfloor x^{\underline{k}} \text { and } x^{\underline{n}}=\sum_{k=0}^{n}(-1)^{n-k}\left\lfloor\begin{array}{l}
n \\
k
\end{array}\right\rfloor x^{\bar{k}}
$$

or

$$
\left\lfloor\begin{array}{l}
n \\
k
\end{array}\right\rfloor=\sum_{j=k}^{n}(-1)^{n-j} s(n, j)\left\{\begin{array}{l}
j \\
k
\end{array}\right\} .
$$

The (unsigned) Lah numbers $\left\lfloor\begin{array}{l}n \\ k\end{array}\right\rfloor$ count the number of partitions of a set of $n$ elements into exactly $k$ ordered lists

$$
\left\lfloor\begin{array}{l}
n \\
k
\end{array}\right\rfloor=\frac{n !}{k !}\left(\begin{array}{l}
n-1 \\
k-1
\end{array}\right) \text { for } n \geq k \geq 1 .
$$

Let $L_{n, k}$ denote the (signed) Lah numbers [2]

$$
L_{n, k}:=(-1)^{n}\left\lfloor\begin{array}{l}
n \\
k
\end{array}\right\rfloor
$$

The exponential generating functions is

$$
\sum_{n \geq k}\left\lfloor\begin{array}{l}
n \\
k
\end{array}\right\rfloor \frac{z^{n}}{n !}=\frac{1}{k !}\left(\frac{z}{1-z}\right)^{k} .
$$

Setting the final sequence $a_{n, 0}=(-1)^{n} B_{n}(x)$ in (2), we get the following matrix

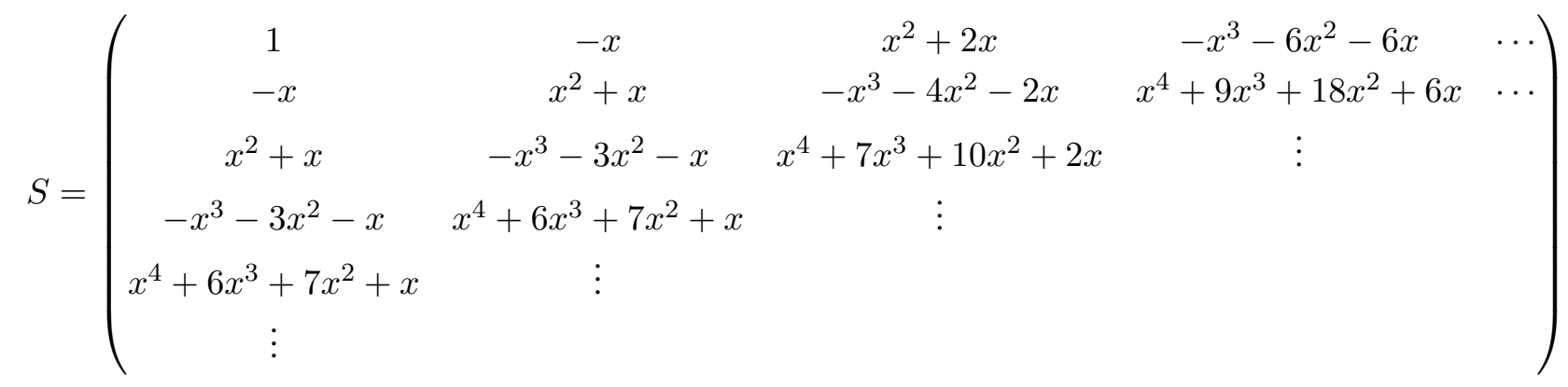


Since

$$
\begin{aligned}
\mathcal{B}_{0}(z) & =\sum_{k \geq 0} B_{k}(x) \frac{(-z)^{k}}{k !} \\
& =\exp \left(x\left(e^{-z}-1\right)\right) .
\end{aligned}
$$

It follows from (5) that the initial sequence has an exponential generating function, given by

$$
\begin{aligned}
\mathcal{A}_{0}(z) & =\mathcal{B}_{0}(\ln (1+z)) \\
& =\exp \left(x\left(\frac{1}{1+z}-1\right)\right) \\
& =\sum_{n \geq 0} L_{n}(x) \frac{z^{n}}{n !},
\end{aligned}
$$

where $L_{n}(x)$ denotes the (signed) Lah polynomials defined by

$$
L_{n}(x):=\sum_{k=0}^{n} L_{n, k} x^{k}
$$

Now, from (6) we have

$$
\begin{aligned}
\sum_{n \geq 0} L_{n}(x) \frac{z^{n}}{n !} & =\exp \left(x\left(\frac{1}{1+z}-1\right)\right) \\
& =\frac{1}{e^{x}}+\frac{1}{e^{x}} \sum_{k \geq 1}\left(\frac{1}{1+z}\right)^{k} \frac{x^{k}}{k !} \\
& =\frac{1}{e^{x}}+\frac{1}{e^{x}}\left(\sum_{k \geq 1}\left(\sum_{n \geq 0}(-1)^{n} \frac{(k-1+n) !}{n !(k-1) !} z^{n}\right) \frac{x^{k}}{k !}\right) \\
& =\frac{1}{e^{x}}+\sum_{n \geq 0}\left(\frac{1}{e^{x}} \sum_{k \geq 0}(-1)^{n} \frac{(k+n) !}{k !(k+1) !} x^{k+1}\right) \frac{z^{n}}{n !}
\end{aligned}
$$

Equating the coefficients of $\frac{z^{n}}{n !}$, we get the Dobiński's formula for the $n$-th $(n \geq 1)$ signed Lah polynomials

$$
L_{n}(x)=\frac{(-1)^{n}}{e^{x}} \sum_{k \geq 0} \frac{(k+n) !}{k !(k+1) !} x^{k+1}, \quad n \geq 1
$$

A variation of Dobiński's formula in terms of Kummer confluent hypergeometric functions is given by

$$
\begin{aligned}
L_{n}(x) & =\frac{(-1)^{n} x}{e^{x}} \sum_{k \geq 0} \frac{(n+1)^{\bar{k}}}{2^{\bar{k}}} \frac{x^{k}}{k !} \\
& =\frac{(-1)^{n} n ! x}{e^{x}}{ }_{1} F_{1}\left(\begin{array}{cc}
n+1 & ; x \\
2 &
\end{array}\right),
\end{aligned}
$$

where ${ }_{1} F_{1}\left(\begin{array}{l}a \\ b\end{array} ; z\right)$ denotes the Kummer confluent hypergeometric functions, defined by $\sum_{n \geq 0} \frac{a^{\bar{n}}}{b^{n}} \frac{z^{n}}{n !}$. 
Theorem 4. For $n, m \geq 0$, we have

$$
\sum_{k=0}^{m} s(m, k)(-1)^{n+k} B_{n+k}(x)=\sum_{k=0}^{n}\left\{\begin{array}{l}
n+m \\
k+m
\end{array}\right\}_{m} L_{m+k}(x) .
$$

If we set $m=0$ in (7), we have

$$
\begin{aligned}
B_{n}(x) & =\sum_{k=0}^{n}\left\{\begin{array}{l}
n \\
k
\end{array}\right\}(-1)^{n} L_{k}(x) \\
& =\frac{x}{e^{x}} \sum_{k=0}^{n}\left\{\begin{array}{l}
n \\
k
\end{array}\right\} n !_{1} F_{1}\left(\begin{array}{cc}
n+1 \\
2
\end{array}\right) .
\end{aligned}
$$

Notice that the Guo-Qi's identity for Bell numbers [4,5] is obtained by setting $x=1$ in (8).

When $n=0$ in (7) we get the inverse Stirling transform for Bell polynomials

$$
L_{m}(x)=\sum_{k=0}^{m} s(m, k)(-1)^{k} B_{k}(x)
$$

Now, setting $n=1$ in (7), we get

$$
\begin{aligned}
\sum_{k=0}^{m} s(m, k)(-1)^{k+1} B_{k+1}(x) & =m L_{m}(x)+L_{m+1}(x) \\
& =\sum_{k=0}^{m}(-1)^{m+1} k !\left(\begin{array}{c}
m \\
k
\end{array}\right)^{2} x^{m-k+1} .
\end{aligned}
$$

\section{An explicit formula for Euler numbers}

The Euler numbers $E_{n}$ can be defined by the exponential generating function

$$
\frac{1}{\cosh z}=\sum_{n \geq 0} E_{n} \frac{z^{n}}{n !} .
$$

It is well-known that $E_{n}$ are a sequence of integers with $E_{2 n+1}=0$ for $n \geq 0$. There are a number of explicit formulae for $E_{n}$, for example (see also [7])

$$
E_{2 n}=i \sum_{k=1}^{2 n+1} \sum_{j=0}^{k} \frac{(-1)^{j}}{2^{k} i^{k} k}\left(\begin{array}{l}
k \\
j
\end{array}\right)(k-2 j)^{2 n+1},
$$

where $i$ denotes the imaginary unit with $i^{2}=-1$. In this section, we propose a new explicit formula for the Euler numbers

$$
E_{n}=-\sum_{k=0}^{n} \frac{k !}{2^{k}}\left\{\begin{array}{l}
n \\
k
\end{array}\right\} \operatorname{Re}\left((i-1)^{k+1}\right)
$$

where $\operatorname{Re}(z)$, denotes the real part of $z$. 
Now, If we take the final sequence $a_{n, 0}=E_{n}$, in (2), we get the following matrix

$$
T=\left(\begin{array}{cccccc}
1 & 0 & -1 & 3 & -6 & \ldots \\
0 & -1 & 1 & 3 & -24 & \ldots \\
-1 & 0 & 5 & -15 & -6 & \ldots \\
0 & 5 & -5 & -51 & 336 & \ldots \\
5 & 0 & -61 & 183 & 714 & \ldots \\
0 & -61 & 61 & 1263 & -7944 & \ldots \\
-61 & 0 & 1385 & -4155 & -35286 & \ldots \\
0 & 1385 & -1385 & -47751 & 294816 & \ldots \\
\vdots & \vdots & \vdots & \vdots & \vdots & \ddots
\end{array}\right) .
$$

It follows from (5) that the initial sequence has an exponential generating function given by

$$
\begin{aligned}
\sum_{n \geq 0} R_{n} \frac{z^{n}}{n !} & =\frac{1}{\cosh (\ln (1+z))} \\
& =\frac{2(1+z)}{2+2 z+z^{2}} .
\end{aligned}
$$

Since $\frac{2(1+z)}{2+2 z+z^{2}}=\frac{1}{z+1-i}+\frac{1}{z+1+i}$, we have

$$
\begin{aligned}
\sum_{n \geq 0} R_{n} \frac{z^{n}}{n !} & =-\sum_{n \geq 0} \frac{1}{2^{n+1}}\left((-1+i)^{n+1}+(-1-i)^{n+1}\right) z^{n} \\
& =-\sum_{n \geq 0} \frac{1}{2^{n}} \operatorname{Re}\left((i-1)^{n+1}\right) z^{n} .
\end{aligned}
$$

Equating the coefficient of $z^{n}$, we get

$$
R_{n}=-\frac{n !}{2^{n}} \operatorname{Re}\left((i-1)^{n+1}\right)
$$

and by the binomial formula we have

$$
R_{n}=\frac{n !}{2^{n}} \sum_{k=0}^{\lfloor(n+1) / 2\rfloor}(-1)^{n-k}\left(\begin{array}{c}
n+1 \\
2 k
\end{array}\right),
$$

where $\lfloor x\rfloor$ denotes the integral part of $x$, that is, the greatest integer not exceeding $x$.

For $n, m \geq 0$, we have

$$
\sum_{k=0}^{m} s(m, k) E_{n+k}=\sum_{k=0}^{n}\left\{\begin{array}{l}
n+m \\
k+m
\end{array}\right\}_{m} R_{m+k} .
$$

Now, setting $m=0$ in (12), we get (9). Putting $n=0$ in (12), we have the following recursive formula Euler numbers involving the Stirling numbers of the first kind

$$
\begin{aligned}
\sum_{k=0}^{m} s(2 m, 2 k) E_{2 k} & =\frac{(2 m) !}{2^{2 m}} \sum_{k=0}^{m}(-1)^{k}\left(\begin{array}{c}
2 m+1 \\
2 k
\end{array}\right) \\
& =\left\{\begin{array}{c}
(-1)^{\left\lfloor\frac{m}{2}\right\rfloor} \frac{(2 m) !}{2^{m}}, m \text { even, } \\
(-1)^{\left\lfloor\frac{m-1}{2}\right\rfloor+1} \frac{(2 m) !}{2^{m}}, m \text { odd. }
\end{array}\right.
\end{aligned}
$$


Thus, for example, when $m=0,1,2,3$, we obtain

$$
\begin{aligned}
E_{0} & =1, \\
E_{2} & =-1, \\
11 E_{2}+E_{4} & =-6, \\
274 E_{2}+85 E_{4}+E_{6} & =90 .
\end{aligned}
$$

\section{Acknowledgements}

The authors would like to thank an anonymous referees for the helpful comments.

\section{References}

[1] Broder, A. Z. (1984) The $r$-Stirling numbers, Discrete Math., 49, 241-259.

[2] Comtet, L. (1974) Advanced Combinatorics. Reidel Publishing Co., Dordrecht.

[3] Graham, R. L., Knuth, D. E., \& Patashnik, O. (1994) Concrete Mathematics. AddisonWesley Publishing Company, Reading, MA.

[4] Guo, B., \& Qi, F. (2014) An explicit formula for Bell numbers in terms of Stirling numbers and hypergeometric functions, Glob. J. Math. Anal., 2, 243-248.

[5] Qi, F. (2016) An explicit formula for the Bell numbers in terms of the Lah and Stirling numbers, Mediterr. J. Math. 13, 2795-2800.

[6] Rahmani, M. (2014) Generalized Stirling transform, Miskolc Math. Notes, 15, 677-690.

[7] Wei, C.-F. \& Qi, F. (2015) Several closed expressions for the Euler numbers. J. Inequal. Appl., 219, 8 pp. 